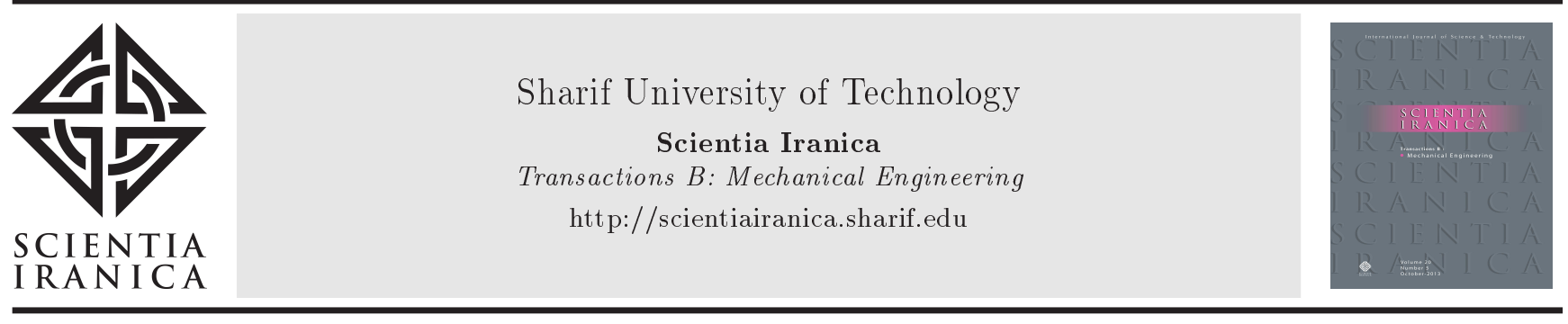

\title{
Closed form solution for direct and inverse kinematics of a US-RS-RPS 2-DOF parallel robot
}

\author{
J. Sanjuan*, D. Serje, and J. Pacheco \\ Department of Mechanical Engineering, Universidad del Norte, Km. 5 Via Puerto Colombia, Barranquilla, Colombia.
}

Received 1 October 2016; received in revised form 1 March 2017; accepted 17 July 2017

\section{KEYWORDS}

Parallel iknematics;

Forward kinematics;

Pose;

Homogeneous

transformation

matrix;

Closed form.

\begin{abstract}
Parallel mechanisms with reduced Degree Of Freedom (DOF) have grown in importance for industry and researchers as they offer a simpler architecture and lower manufacturing/operating costs with great performance. In this paper, a two-degree-offreedom parallel robot is proposed and analyzed. The robot with a fixed base, a moving platform, and three legs achieve translational and rotational motions through actuation on prismatic and revolute joints and can be applied to pick-and-place applications, vehicle simulators, etc. Through homogeneous transformation matrices and Sylvesters dialytic elimination method, a closed-form solution for direct kinematics is obtained for all possible assembly modes. Inverse kinematics is solved by the closed-form solution as well. This greatly decreases computational time, suggesting the optimality of the proposed approach. A case study is investigated to validate the solutions found and is compared with a CAD model to corroborate the obtained results. Finally, a workspace calculation is carried out for different geometrical parameters of the robot.

(C) 2018 Sharif University of Technology. All rights reserved.
\end{abstract}

\section{Introduction}

Parallel Kinematic Machines (PKMs), compared to serial robots, offer some useful features such as higher structural rigidity (stiffness), kinematic accuracy (noncumulative joint error), higher payload to robot weight ratio, compactness, and modularity [1-3]. In the past two decades, all of these advantages have won PKMs special reverence for the industry in the fields of machine tooling, high-speed pick-and-place applications, vehicle driving simulators, and solar tracking mechanisms among others. One of the main issues of PKMs is their complex forward kinematics, often implying to find the solution of nonlinear systems of equations which may not be unique [4] and their

*. Corresponding author.

E-mail address: jdecaro@uninorte.edu.co (J. Sanjuan)

doi: $10.24200 /$ sci.2017.4341

limited workspace which limits their application in some industry markets [5].

In literature, forward kinematics has received extensive attention. Therefore, many approaches have been proposed, classified into two main classes: numerical methods and analytic techniques. Different numerical methods have been applied, e.g., neural networks strategies [6], Taylor expansion series with $n$-order polynomials [7], Newton classical methods [3], and fuzzy inference systems [8]. Although numerical techniques have successfully achieved a fast solution to some problems, their accuracy is dependent on iterations required for a good convergence [9]; they fail to describe the set of solutions to the nonlinear equations governing the problem [10]. Other numerical/graphical methods use CAD functionalities to design computer simulation mechanisms of PKMs that can be used to analyze forward kinematics; further, a variation geometry approach is proposed to shape and solve the reachable workspace problem [11].

To find all the forward kinematics solutions for 


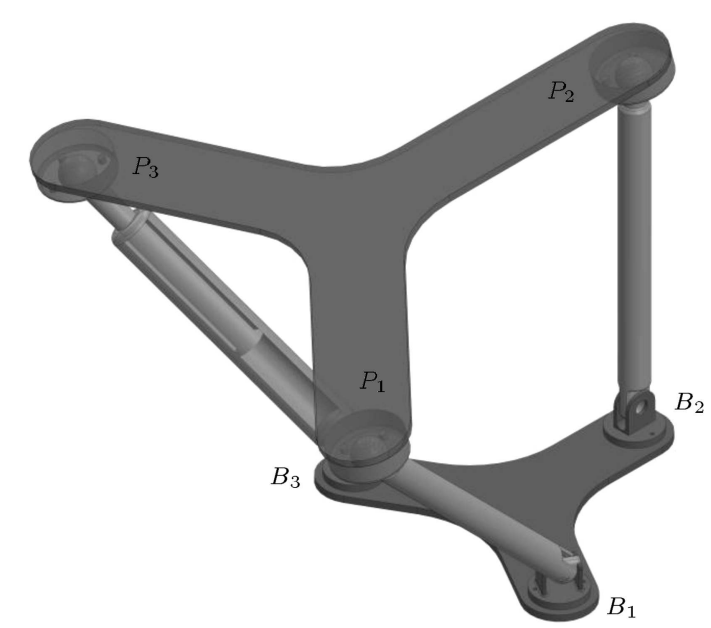

Figure 1. The US-RS-RPS parallel robot.

different configurations on PKMs, various analytical techniques have been used for different requirements, e.g., Sylvesters dialytic elimination method on a 3 (RSS)-S fully spherical robot [12], homotopy continuation method for a 3-UPU translational parallel robot [13], and Grobner basis for lexicographic ordering of equations for a planar Stewart platform [14]. Although analytical strategies lead to the discovery of a closed-form solution, its complexity often requires the use of numerical methods; for instance, Dhingra et al. found a 20th-degree polynomial for the direct kinematics of the Stewart platform [15].

To overcome complex kinematics and control, designers have explored different architectures from universal full mobility parallel robots, such as the Gough-Stewart platform, to robots with reduced DOF with a simpler architecture and lower manufacturing/operating costs. Although decreasing the DOF reduces the available workspace, it also lowers complexity on forward kinematics solution, incidence of singularities, voids and legs collisions [16] from the tracking space as evidenced by Dunlop et al. [17]. In particular, the two DOF parallel mechanisms have attracted much attention of the designers, and various examples of applications of two spatial and planar DOFs mechanisms can be found in different industrial sectors; for instance, Zhang et al. described a 2-DOF mechanism in a vehicle driving simulator [18]; Cammarata designed a 2-DOF mechanism for solar tracking systems [19]; Rico et al. developed a knee rehabilitation device using a planar parallel mechanism [20]. Although there is an increased interest in those mechanisms, there are still many types that have not been analyzed.

This paper studies a US-RS-RPS parallel robot, which is a 2-DOF parallel robot with translational and rotational capabilities (shown in Figure 1). This architecture offers simple kinematic actuation on prismatic and revolute joints and can be used on pickand-place applications, simple vehicle driving simu- lators, solar tracking mechanisms or others according to users' requirements. The position analysis of this mechanism is carried out using homogeneous transformation matrices. These matrices are mainly used for analysis of serial mechanisms, allowing for an intuitive understanding of the relationship between passive and active joints and the position and orientations of moving platform [21]. A closed-form solution for all configurations is achieved using the Sylvesters dialytic elimination method. Inverse kinematics is also analyzed; finally, a case study is shown with a symmetric structure exhibiting four real configurations, and the workspace calculated is also done for illustration purposes.

\section{Description of the US-RS-RPS parallel robot}

The US-RS-RPS parallel robot is composed of a moving platform $\left(P_{1}, P_{2}, P_{3}\right)$, a fixed base $\left(B_{1}, B_{2}, B_{3}\right)$, and three legs $\left(B_{1} P_{1}, B_{2} P_{2}, B_{3} P_{3}\right)$ (see Figures 1 and 2 ). Leg one has a universal (U) joint (passive) attached to the base and is attached to the moving platform by a spherical (S) joint; the universal joint is described by angles $\psi$ and $\theta$. Leg two has an actuated revolute (R) joint assembled to the base and a spherical $(S)$ joint attached to the moving platform; angle $\beta$ describes the revolute joint. Leg three has a revolute $(R)$ joint connected to the base, an actuated prismatic $(\mathrm{P})$ joint, and a spherical joint connected to the moving platform; angle $\psi$ describes the revolute joint and $\rho$ describes the prismatic actuation as an expansion/contraction of the leg's initial length. The length of the legs is defined by $L$.

Each leg is attached to the base at points $B_{i}$ and to the moving platform at points $P_{i}$, as shown in Figures 2 and 3 . Reference coordinate system $x y z$, is attached to the center of the fixed base, $O$. $B_{i}$ points are referenced to coordinate system $x y z$ through a translation in $z$ axis described by distance $d$ followed by a rotation along $x$ axis, measured through angle $\lambda_{i}$. The orientation of the moving platform is represented by coordinate system $u v w$ which is located at the center of the moving platform at point $O_{p} . P_{i}$ points are referenced to the coordinate system uvw through a translation in $w$ axis described by distance $a$ followed by a rotation along $u$ axis, measured through angle $\gamma_{i}$. Coordinate system uvw and its position are described by homogeneous transformation matrix $M_{O}^{O_{p}}$. Coordinate system $u v w$ is defined by roll, pitch and yaw angle parameters, namely a rotation of $\alpha_{x}$ about fixed $x$ axis, followed by rotation $\alpha_{y}$ about fixed $y$ axis, and rotation $\alpha_{z}$ about fixed $z$ axis. There is no particular reason for selecting such a definition. Thus, matrix $M_{O}^{O_{p}}$ can be expressed by Eq. (1) as shown in Box I. 


$$
\boldsymbol{M}_{\boldsymbol{O}}^{\boldsymbol{O}_{p}}=\left[\begin{array}{cccc}
c \alpha_{y} c \alpha_{z} & -c \alpha_{y} s \alpha_{z} & s \alpha_{y} & O_{x} \\
c \alpha_{x} s \alpha_{z}+c \alpha_{z} s \alpha_{x} s \alpha_{y} & c \alpha_{x} c \alpha_{z}-s \alpha_{x} s \alpha_{y} s \alpha_{z} & -c \alpha_{y} s \alpha_{x} & O_{y} \\
s \alpha_{x} s \alpha_{z}-c \alpha_{x} c \alpha_{z} s \alpha_{y} & c \alpha_{z} s \alpha_{x}+c \alpha_{x} s \alpha_{y} s \alpha_{z} & c \alpha_{x} c \alpha_{y} & O_{z} \\
0 & 0 & 0 & 1
\end{array}\right]
$$

Box I

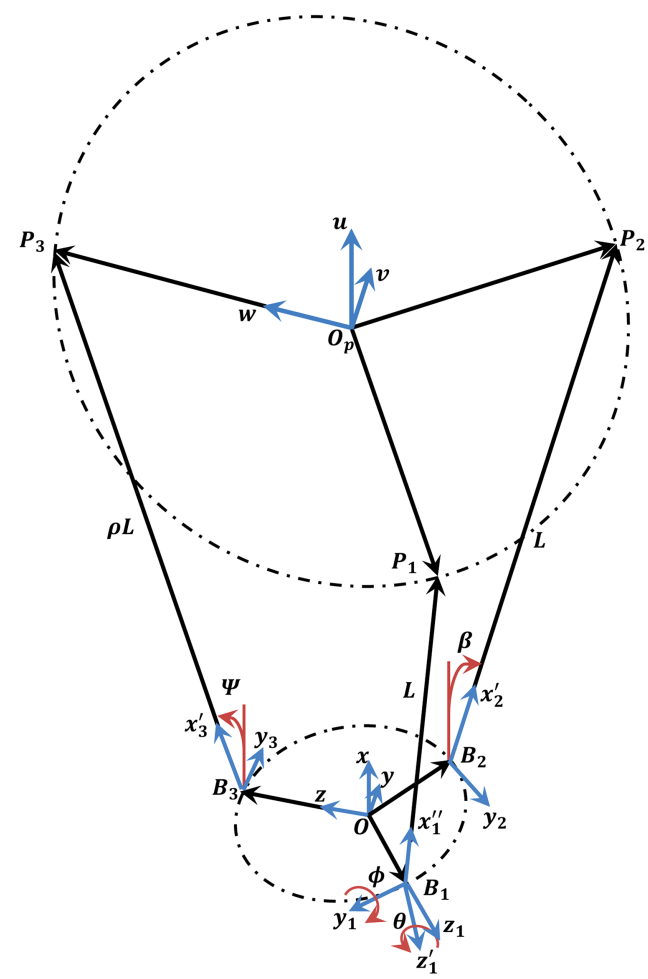

Figure 2. Schematic diagram of the parallel manipulator.

$\cos (*)$ and $\sin (*)$ are represented by $c(*)$ and $s(*)$. Eq. (1) is written in terms of the unit vectors uvw attached to platform and its origin as follows:

$$
M_{\boldsymbol{O}}^{\boldsymbol{O}_{p}}=\left[\begin{array}{cccc}
\overrightarrow{\boldsymbol{u}} & \overrightarrow{\boldsymbol{v}} & \overrightarrow{\boldsymbol{w}} & \overrightarrow{\boldsymbol{O}_{p}} \\
0 & 0 & 0 & 1
\end{array}\right]
$$

The center of the moving platform can be represented as a function of $P_{i}$ points through the barycenter equation:

$$
\overrightarrow{O_{p}}=\frac{1}{3} \sum_{i=1}^{3} P_{i}
$$

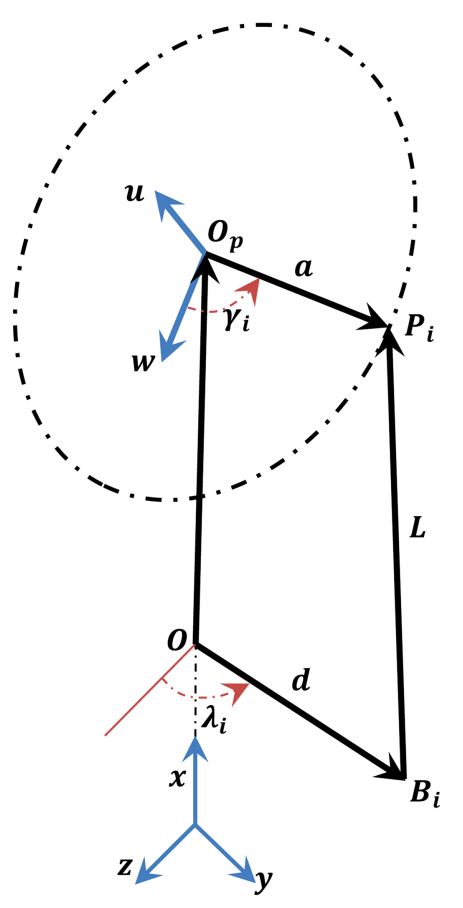

Figure 3. Geometry of a general kinematic chain.

Considering that the direction of unit vector $\overrightarrow{\boldsymbol{w}}$ is along vector $\overrightarrow{O_{p} P_{3}}$, the direction of unit vector $\overrightarrow{\boldsymbol{u}}$ is perpendicular to the moving platform, and unit vector $\overrightarrow{\boldsymbol{v}}$ is perpendicular to unit vectors $\overrightarrow{\boldsymbol{u}}$ and $\overrightarrow{\boldsymbol{w}}$, then Eq. (2) is written in terms of $P_{i}$ points as shown in Box II. Using the results from Eqs. (2) and (4), the rotation angles of Eq. (1) can be found in terms of $P_{i}$ points using the relationship shown below:

$$
\begin{aligned}
& \alpha_{y}=a \tan 2\left(\left(u_{x}^{2}+v_{x}^{2}\right)^{\frac{1}{2}}, w_{x}\right), \\
& \alpha_{z}=a \tan 2\left(\frac{-v_{x}}{c \alpha_{y}}, \frac{u_{x}}{c \alpha_{y}}\right),
\end{aligned}
$$

$$
\boldsymbol{M}_{\boldsymbol{O}}^{O_{p}}=\left[\begin{array}{cccc}
\overrightarrow{O_{p} P_{i}} \times \overrightarrow{O_{p} P_{i+1}} & \overrightarrow{O_{p} P_{3}} \times\left(\overrightarrow{O_{p} P_{i}} \times \overrightarrow{O_{p} P_{i+1}}\right) \\
\left\|\overrightarrow{O_{p} P_{1}} \times \overrightarrow{O_{p} P_{2}}\right\| & \frac{\overrightarrow{O_{p} P_{3}}}{\overrightarrow{O_{p} P_{1}} \times \overrightarrow{O_{p} P_{2}} \|} & \overrightarrow{O_{p} P_{3} \|} & \overrightarrow{O_{p}} \\
0 & 0 & 0 & 1
\end{array}\right]
$$




$$
\alpha_{x}=a \tan 2\left(\frac{-w_{x}}{c \alpha_{y}}, \frac{w_{z}}{c \alpha_{y}}\right) .
$$

Finally, the DOFs of the US-RS-RPS parallel robot are calculated using both the Grübler-Kutzbach equation as used in [22] and the analytical DOF method of an end effector using reciprocal screw theory is depicted in [23]. First, using the Grübler-Kutzbach yields:

$$
M=\xi(L-1)-\sum_{i=1}^{g} f_{i}=6(7-1)-34=2,
$$

where $L$ represents the number of links, $\xi$ represents the task space, $f_{i}$ denotes the DOF of joint $i$, and $g$ represents the number of joints. The coordinate system attached to the center of the moving platform can be oriented and displaced by the actuation of prismatic joint, $\rho$, and revolute joint, $\beta$.

The second approach calculates twist $\left(\$_{i}\right)$ of each leg of the platform and uses these results to compute the wrench $\left(\$_{i}^{r}\right)$ of each leg through the reciprocal screws formula presented below:

$$
\$_{i} \odot \$_{i}^{r}=0 .
$$

The wrenches obtained are grouped in matrix $\$^{r}$, and the DOFs of the mechanism are solved using again the reciprocal screws formula, only this time to obtain the value of $\$^{F}$ as shown below:

$$
\$^{F} \odot \$_{i}^{r}=0 \text {. }
$$

The non-zero rows of matrix $\$^{F}$ represent the DOF of the mechanism; for the case of the US-RS-RPS parallel robot, the analyses are carried out obtaining two rows for matrix $\$^{F}$, and then the mechanism has two DOFs.

\section{Direct kinematic analysis}

The US-RS-RPS parallel robot, as mentioned before, has two DOFs, assuming that the actuation falls on prismatic $(\rho)$ and revolute joint on the second leg $(\beta)$. There are still three passive joints which need to be solved in order to solve the direct kinematic problem; these passive joints are $\phi, \theta$, and $\psi$.

To solve the direct kinematics primarily, coupling points, $B_{i}$, are obtained using two transformations. First, considering a rotation in the direction of $x$ axis by angle $\lambda_{i}$ and a displacement in $z$ axis by distance $d$, these two transformations yield the homogeneous transformation matrix shown below:

$$
\boldsymbol{T}_{\boldsymbol{B}_{\boldsymbol{i}}}=\left[\begin{array}{cccc}
1 & 0 & 0 & 0 \\
0 & c \lambda_{i} & -s \lambda_{i} & -d s \lambda_{i} \\
0 & s \lambda_{i} & c \lambda_{i} & d c \lambda_{i} \\
0 & 0 & 0 & 1
\end{array}\right]
$$

$$
=\left[\begin{array}{cccc}
\overrightarrow{\boldsymbol{x}_{l}} & \overrightarrow{\boldsymbol{y}_{\boldsymbol{l}}} & \overrightarrow{\boldsymbol{z}_{l}} & \overrightarrow{\boldsymbol{B}_{\boldsymbol{i}}} \\
0 & 0 & 0 & 1
\end{array}\right]
$$

where $\overrightarrow{x_{i}}, \overrightarrow{y_{i}}$, and $\overrightarrow{z_{i}}$ are the directions of the coordinate system attached to point $B_{i}$.

Furthermore, according to the representation shown in Figure 3, the position equation associated with $P_{i}$ points can be defined in two ways as shown in the following equations:

$$
\begin{aligned}
& P_{i}=O_{p}+O_{p} P_{i}, \\
& P_{i}=B_{i}+B_{i} P_{i} .
\end{aligned}
$$

These equations can also be stated in terms of the homogeneous transformation matrix. For Eq. (12), the position of $O_{p}$ is obtained using the homogeneous transformation matrix, $\boldsymbol{M}_{\boldsymbol{O}}^{\boldsymbol{O}_{p}}$; additionally, position vector $\overrightarrow{O_{p} P_{i}}$ is determined by two transformations: a rotation in the direction of $u$ axis by angle $\gamma_{i}$, and a displacement in "the rotated" $w$ axis by distance $a$, obtaining subsequent equivalence for $P_{i}$ :

$$
\begin{aligned}
& P_{i}=\left[\begin{array}{l}
O_{x} \\
O_{y} \\
O_{z}
\end{array}\right] \\
& +\left[\begin{array}{c}
a c \gamma_{i} s \alpha_{y}+a c \alpha_{y} s \alpha_{z} s \gamma_{i} \\
-a s \gamma_{i}\left(c \alpha_{x} c \alpha_{z}-s \alpha_{x} s \alpha_{y} s \alpha_{z}\right)-a c \alpha_{y} c \gamma_{i} s \alpha_{x} \\
a c \alpha_{x} c \alpha_{y} c \gamma_{i}-a s \gamma_{i}\left(c \alpha_{z} s \alpha_{x}+c \alpha_{x} s \alpha_{y} s \alpha_{z}\right)
\end{array}\right](14)
\end{aligned}
$$

Besides, the solution to Eq. (15) is achieved by successive transformations through the axis of each joint linked to every arm of the mechanism generating the coordinates of every $P_{i}$ point in the robot. As depicted below:

$$
\begin{aligned}
& P_{1}=\left[\begin{array}{c}
L c \phi c \theta \\
L\left(c \lambda_{1} s \phi+c \phi s \lambda_{1} s \theta\right)-d s \lambda_{1} \\
L\left(s \lambda_{1} s \phi-c \phi c \lambda_{1} s \theta\right)+d c \lambda_{1}
\end{array}\right], \\
& P_{2}=\left[\begin{array}{c}
L c \beta \\
-s \lambda_{2}(d-L s \beta) \\
c \lambda_{2}(d-L s \beta)
\end{array}\right] \\
& P_{3}=\left[\begin{array}{c}
\rho L c \psi \\
-s \lambda_{3}(d-L s \psi) \\
c \lambda_{3}(d-L s \psi)
\end{array}\right] .
\end{aligned}
$$

Using the results of Eqs. (15), (16), and (17), the next three equations express the distance of segments $\overline{P_{1} P_{2}}, \overline{P_{2} P_{3}}$, and $\overline{P_{1} P_{3}}$, respectively:

$$
K_{1}+K_{2} c \phi s \theta+K_{3} c \phi c \theta+K_{4} s \phi=0
$$




$$
\begin{aligned}
& K_{5}+K_{6} c \psi+K_{7} s \psi=0 \\
& K_{8}+K_{9} c \phi s \theta+K_{10} c \phi c \theta+K_{11} s \phi=0
\end{aligned}
$$

where terms $K_{1}$ to $K_{7}$ are dependent on geometric parameters (dimensions), $d, L_{1}, P_{1} P_{2}, P_{2} P_{3}, P_{1} P_{3}$, $\lambda_{1}, \lambda_{2}, \lambda_{3}$, and active joints, $\beta$ and $\rho$; terms $K_{8}$ to $K_{11}$ are functions of $\psi$ and the mentioned parameters. The detailed expressions for $K_{1}$ to $K_{11}$ terms are given in the Appendix. Eq. (19) relates passive joint $\psi$ with active joint $\beta$ implicitly; to explicitly solve this equation for $\psi$, an half-angle substitution is used, resulting in:

$$
\psi=2 \tan ^{-1}\left(\frac{-K_{7} \pm \sqrt{K_{7}^{2}-K_{5}^{2}+K_{6}^{2}}}{K_{5}-K_{6}}\right) .
$$

Using the results of $\psi$ on Eqs. (18) and (20) and performing another half angle substitution for angle $\phi$ on both equations yield:

$$
\begin{aligned}
& K_{12} T_{\phi}^{2}+K_{13} T_{\phi}+K_{14}=0, \\
& K_{15} T_{\phi}^{2}+K_{16} T_{\phi}+K_{17}=0,
\end{aligned}
$$

where $T$ stands for half angle substitution, $T_{\phi}=$ $\tan (\phi / 2)$; similarly, for Eq. (21), coefficients $K_{12}$ to $K_{17}$ are functions of the mentioned geometric parameters, active joints $\beta$ and $\rho$ and passive joints $\theta$ and $\psi$. The detailed expressions for $K_{12}$ to $K_{17}$ coefficients are given in the Appendix. The coefficients of Eqs. (22) and (23) are used to create the matrix shown below:

$$
S=\left[\begin{array}{cccc}
0 & K_{12} & K_{13} & K_{14} \\
K_{12} & K_{13} & K_{14} & 0 \\
0 & K_{15} & K_{16} & K_{17} \\
K_{15} & K_{16} & K_{17} & 0
\end{array}\right]
$$

For this matrix, if $T_{\phi}$ is a solution to Eqs. (22) and (23), then coefficients $K_{12}, K_{13}$, and $K_{14}$ are linear to $K_{15}, K_{16}$, and $K_{17}$; as a consequence, the determinant of matrix $S$ must be equal to zero, which produces the equation below:

$$
\begin{aligned}
\operatorname{det}(S)= & K_{12}^{2} K_{17}^{2}-K_{12} K_{13} K_{16} K_{17} \\
& -2 K_{12} K_{14} K_{15} K_{17}+K_{12} K_{14} K_{16}^{2} \\
& +K_{13}^{2} K_{15} K_{17}-K_{13} K_{14} K_{15} K_{16} \\
& +K_{14}^{2} K_{15}^{2}=0
\end{aligned}
$$

As mentioned, coefficients $K_{12}$ to $K_{17}$ are functions of already known parameters, except for $\theta$, which is the only unknown parameter in this equation. To solve Eq. (18) for $\theta$, another half angle substitution is carried out, yielding the next quartic function:

$$
K_{18} T_{\theta}^{4}+K_{19} T_{\theta}^{3}+K_{20} T_{\theta}^{2}+K_{21} T_{\theta}+K_{22}=0,
$$

where $T_{\theta}$ represents half angle substitution $T_{\theta}=$ $\tan (\theta / 2)$. Coefficients $K_{18}$ to $K_{22}$ are described in detail in the Appendix. Eq. (26) has four solutions and many analytical methods exist to solve it [24]. Once a solution for $\theta$ is obtained, it is easy to solve $\phi$ using one of Eqs. (22) and (23) and substituting $T_{\phi}=\tan (\phi / 2)$. Using Eq. (22) yields:

$$
\phi=2 \tan ^{-1}\left(\frac{-K_{13} \pm \sqrt{K_{13}^{2}-4 K_{12} K_{14}}}{2 K_{12}}\right) .
$$

Once the passive joints are explicitly solved, the direct kinematic is obtained in a straightforward way, as every coupling point of the platform is a function of the passive and active joints as depicted in Eqs. (15) to (17). Then, as described in Eqs. (3) to (7), the position and orientation of the moving platform is also found as these equations are functions of the coupling points.

\section{Inverse kinematics}

The inverse kinematic problem focuses on the solution of the active joints position, knowing the position and orientation of the moving platform. As mentioned before, the position and orientation of the moving platform are described by Eq. (1). Moreover, Eq. (15) describes the position of $P_{i}$ points as a function of the orientation and the position of the moving platform. Using these results, the inverse kinematic problem is solved obtaining the values of actives joints, $\beta$ and $\rho$, as functions of points $P_{1}, P_{2}$, and $P_{3}$.

Employing the expression for $x$ coordinate of point $P_{2}$ and dividing it by the expression for $y$ coordinate of point $P_{2}$ easily yield the expression for active joint $\beta$ :

$$
\beta=\tan ^{-1}\left(\frac{P_{2 y}+d s \lambda_{2}}{s \lambda_{2} P_{2 x}}\right) .
$$

To find the solution for active joint initially, it is needed to solve the passive joint, $\psi$. Using a similar procedure rather than the used one to find $\beta, \psi$ is found using the expression for $x$ coordinate of point $P_{3}$ and the expression for $y$ coordinate of point $P_{3}$, producing:

$$
\psi=\tan ^{-1}\left(\frac{P_{3 y}+d s \lambda_{3}}{P_{3 x}}\right) .
$$

At last, the solution for active joint $\rho$ is found in a straightforward way replacing the obtained value in Eq. (22) in the expression for $z$ coordinate of point $P_{3}$, generating:

$$
\rho=\frac{c \lambda_{3} d-P_{3}}{L_{1} s \psi} .
$$

Eqs. (14), (28), (29), and (30) solve the inverse kinematic for the US-RS-RPS parallel robot. 


\section{Case study}

In this section, an example of a solution to direct kinematic problem of the US-RS-RPS parallel robot is presented. For this purpose, the constant geometric parameters $\left(d, L_{1}, a, \lambda_{1}, \lambda_{2}, \lambda_{3}\right)$ and position of the actuators $(\beta, \rho)$ are supplied. The solution will determine the position of the coupling points of the platform $\left(P_{1}, P_{2}, P_{3}\right)$ by obtaining three angles $\phi, \theta$, and $\psi$. Afterwards, in order to validate the solution, the direct kinematic solution is used as an input to the inverse kinematic model.

The architecture parameters and actuation values are shown in Table $1 . K_{i}$ coefficients used to solve the forward kinematic problem are also depicted for every solution (assembly modes) in Table 2. Using these values, passive joints $\phi, \theta$, and $\psi$ are calculated, the values are listed in Table 3 , and the coupling points $\left(P_{1}, P_{2}, P_{3}\right)$ are also listed in Table 3 . These parameters show the particular solution for each assembly mode. According to these results, the solutions are shown graphically in Figure 4.

Validation of the proposed model is made using the coupling points as inputs for the inverse kinematic model, and then the actives joints are obtained, as shown in Table 4. The results corroborate the accuracy of the proposed model for the direct position analysis of the manipulator. This is also verified through a comparison with a $3 \mathrm{D}$ CAD model developed with proper constraints and dimensions to solve the kinematic parameters of the platform, achieving the same results from the proposed closed-form solution [25]. Figure 5 shows an example CAD model used for assembly mode 3 evaluation.

\section{Workspace calculation}

For the current case study, only theoretical workspace is considered and calculated using the solution obtained by forward kinematics [26]. For assembly mode C (see Figure 4), with applied geometrical and joint constraints, workspace is calculated as a radial projection of platform center on a sphere in Cartesian space, as shown in Figure 6. By using a projected workspace, translational and rotational capabilities of manipulator can be seen in one graph. As stated before, robot configuration and dimensioning is crucial; therefore, different topologies and characteristics can be achieved through variations on geometrical parameters. In Figure 6(a), the dimensions of the robot are used for the case of study, and this configuration yields a noncontinuous workspace divided into three zones, imply-

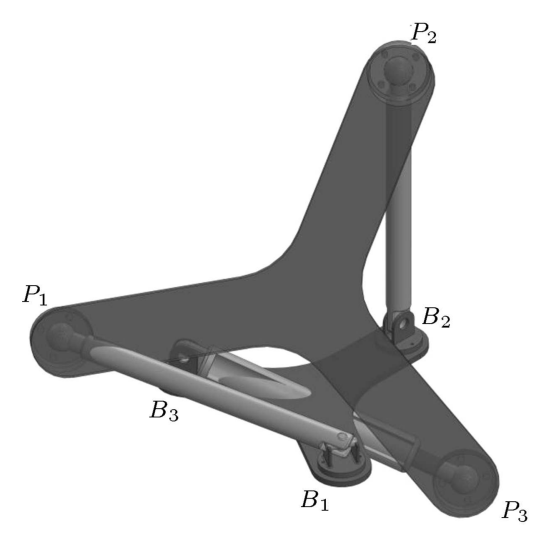

(a)

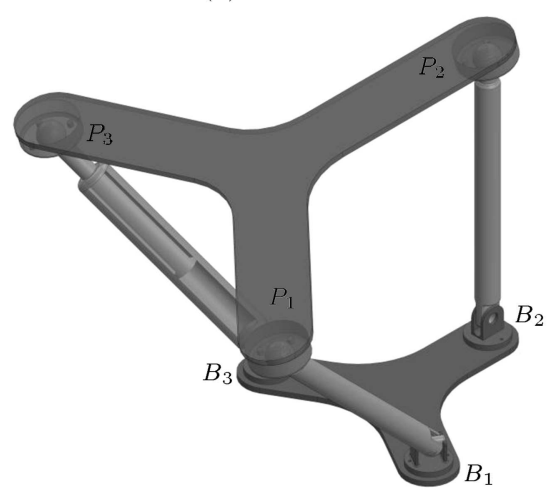

(c)

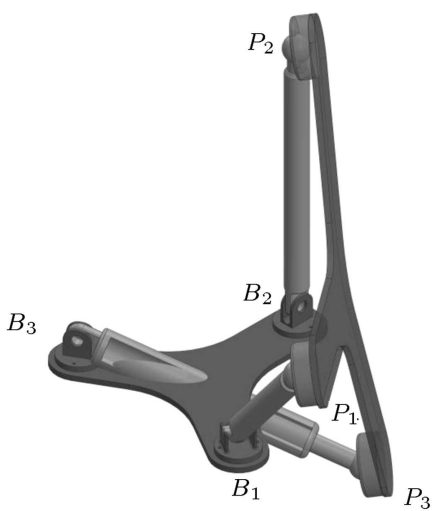

(b)

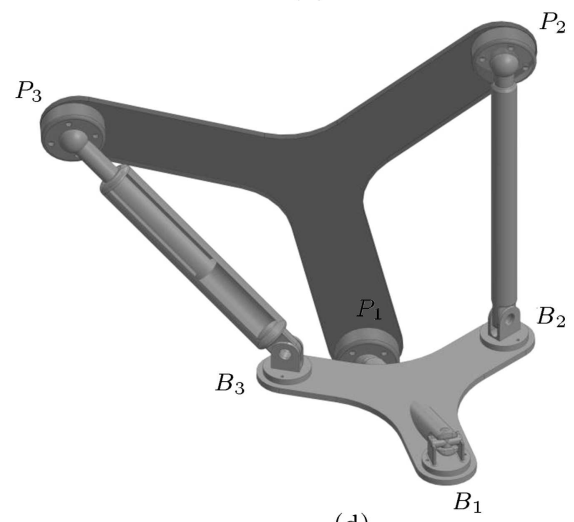

(d)

Figure 4. Assembly modes. 
Table 1. Input data for the US-RS-RPS parallel robot case study.

\begin{tabular}{cccccccc}
\hline $\boldsymbol{d}(\mathbf{m m})$ & $\boldsymbol{L}(\mathbf{m m})$ & $\boldsymbol{a}(\mathbf{m m})$ & $\boldsymbol{\lambda}_{\mathbf{1}}$ and $\boldsymbol{\gamma}_{\mathbf{1}}$ & $\boldsymbol{\lambda}_{\mathbf{2}}$ and $\boldsymbol{\gamma}_{\mathbf{2}}$ & $\boldsymbol{\lambda}_{\mathbf{3}}$ and $\boldsymbol{\gamma}_{\mathbf{3}}$ & $\boldsymbol{\beta}$ & $\boldsymbol{\rho}$ \\
\hline 40 & 96 & 78.46 & $120^{\circ}$ & $-120^{\circ}$ & $0^{\circ}$ & $0^{\circ}$ & 1 \\
\hline
\end{tabular}

Table 2. Coefficients $K_{i}$ for all assembly modes.

\begin{tabular}{ccccc}
\hline & \multicolumn{4}{c}{ Assembly mode } \\
\cline { 2 - 5 } $\mathbf{1}$ & $\mathbf{2}$ & $\mathbf{3}$ & $\mathbf{4}$ \\
\hline$K_{1}$ & $4.763 \times 10^{3}$ & $4.763 \times 10^{3}$ & $4.763 \times 10^{3}$ & $4.763 \times 10^{3}$ \\
$K_{2}$ & $-1.152 \times 10^{4}$ & $-1.152 \times 10^{4}$ & $-1.152 \times 10^{4}$ & $-1.152 \times 10^{4}$ \\
$K_{3}$ & $-1.843 \times 10^{4}$ & $-1.843 \times 10^{4}$ & $-1.843 \times 10^{4}$ & $-1.843 \times 10^{4}$ \\
$K_{4}$ & $6.651 \times 10^{3}$ & $6.651 \times 10^{3}$ & $6.651 \times 10^{3}$ & $6.651 \times 10^{3}$ \\
$K_{5}$ & $4.763 \times 10^{3}$ & $4.763 \times 10^{3}$ & $4.763 \times 10^{3}$ & $4.763 \times 10^{3}$ \\
$K_{6}$ & $-1.843 \times 10^{4}$ & $-1.843 \times 10^{4}$ & $-1.843 \times 10^{4}$ & $-1.843 \times 10^{4}$ \\
$K_{7}$ & $-1.152 \times 10^{4}$ & $-1.152 \times 10^{4}$ & $-1.152 \times 10^{4}$ & $-1.152 \times 10^{4}$ \\
$K_{8}$ & $-6.106 \times 10^{3}$ & $-6.106 \times 10^{3}$ & $1.296 \times 10^{4}$ & $1.296 \times 10^{4}$ \\
$K_{9}$ & $-2.824 \times 10^{3}$ & $-2.824 \times 10^{3}$ & $-1.807 \times 10^{4}$ & $-1.807 \times 10^{4}$ \\
$K_{10}$ & $6.106 \times 10^{3}$ & $6.106 \times 10^{3}$ & $-1.296 \times 10^{4}$ & $-1.296 \times 10^{4}$ \\
$K_{11}$ & $8.410 \times 10^{3}$ & $8.410 \times 10^{3}$ & $-1.800 \times 10^{4}$ & $-1.800 \times 10^{4}$ \\
$K_{12}$ & $2.119 \times 10^{4}$ & $9.387 \times 10^{3}$ & $1.555 \times 10^{4}$ & $9.581 \times 10^{3}$ \\
$K_{13}$ & $1.330 \times 10^{4}$ & $1.330 \times 10^{4}$ & $1.330 \times 10^{4}$ & $1.330 \times 10^{4}$ \\
$K_{14}$ & $-1.166 \times 10^{4}$ & $1.397 \times 10^{2}$ & $-6.023 \times 10^{3}$ & $-5.493 \times 10^{1}$ \\
$K_{15}$ & $-5.200 \times 10^{3}$ & $-1.239 \times 10^{4}$ & $1.582 \times 10^{4}$ & $2.577 \times 10^{4}$ \\
$K_{16}$ & $1.682 \times 10^{4}$ & $1.682 \times 10^{4}$ & $-3.601 \times 10^{4}$ & $-3.601 \times 10^{4}$ \\
$K_{17}$ & $-7.013 \times 10^{3}$ & $.793 \times 10^{2}$ & $1.010 \times 10^{4}$ & $1.478 \times 10^{2}$ \\
$K_{18}$ & $-9.918 \times 10^{16}$ & $-9.918 \times 10^{16}$ & $-4.553 \times 10^{17}$ & $-4.553 \times 10^{17}$ \\
$K_{19}$ & $1.325 \times 10^{17}$ & $1.325 \times 10^{17}$ & $2.012 \times 10^{18}$ & $2.012 \times 10^{18}$ \\
$K_{20}$ & $3.173 \times 10^{17}$ & $3.173 \times 10^{17}$ & $-2.700 \times 10^{17}$ & $-2.700 \times 10^{17}$ \\
$K_{21}$ & $-1.325 \times 10^{17}$ & $-1.325 \times 10^{17}$ & $-2.012 \times 10^{18}$ & $-2.012 \times 10^{18}$ \\
$K_{22}$ & $-9.918 \times 10^{16}$ & $-9.918 \times 10^{16}$ & $-4.553 \times 10^{17}$ & $-4.553 \times 10^{17}$ \\
\hline & & & &
\end{tabular}

Table 3. Solutions of the case study.

\begin{tabular}{|c|c|c|c|c|c|c|}
\hline A. mode & $\phi$ & $\theta$ & $\varphi$ & $P_{1}$ & $P_{2}$ & $P_{3}$ \\
\hline 1 & $109.349^{\circ}$ & $72.92^{\circ}$ & $52.363^{\circ}$ & {$\left[\begin{array}{c}17.22 \\
-24.12 \\
73.856\end{array}\right]$} & $\begin{array}{c}96 \\
34.641 \\
-20\end{array}$ & {$\left[\begin{array}{c}-31.804 \\
0 \\
-50.579\end{array}\right]$} \\
\hline 2 & $109.349^{\circ}$ & $-45.710^{\circ}$ & $-1.215^{\circ}$ & {$\left[\begin{array}{c}67.02 \\
-93.13 \\
-56.112\end{array}\right]$} & $\begin{array}{c}96 \\
34.641 \\
-20\end{array}$ & {$\left[\begin{array}{c}-31.804 \\
0 \\
-50.579\end{array}\right]$} \\
\hline 3 & $-45.338^{\circ}$ & $-28.241^{\circ}$ & $36.263^{\circ}$ & {$\left[\begin{array}{c}68.191 \\
-94.75 \\
10.86\end{array}\right]$} & $\begin{array}{c}96 \\
34.641 \\
-20\end{array}$ & {$\left[\begin{array}{c}67.483 \\
0 \\
108.28\end{array}\right]$} \\
\hline 4 & $-45.338^{\circ}$ & $109.2^{\circ}$ & $0.469^{\circ}$ & {$\left[\begin{array}{c}-31.57 \\
43.48 \\
26.01\end{array}\right]$} & $\begin{array}{c}96 \\
34.641 \\
-20\end{array}$ & {$\left[\begin{array}{c}67.483 \\
0 \\
108.28\end{array}\right]$} \\
\hline
\end{tabular}


Table 4. Solutions of the inverse kinematics problem for the assembly modes.

\begin{tabular}{|c|c|c|c|c|c|}
\hline \multirow[t]{2}{*}{ A. mode } & \multicolumn{3}{|c|}{ Coupling point $\left[P_{1}, P_{2}, P_{3}\right]$} & \multirow[t]{2}{*}{$\boldsymbol{\beta}$} & \multirow[t]{2}{*}{$\rho$} \\
\hline & {$[17.22$} & 96 & $-31.804]$ & & \\
\hline \multirow[t]{3}{*}{1} & -24.12 & 34.641 & 0 & $0^{\circ}$ & 1 \\
\hline & 73.856 & -20 & -50.579 & & \\
\hline & 67.02 & 96 & -31.804 & & \\
\hline \multirow[t]{3}{*}{2} & -93.13 & 34.641 & 0 & $0^{\circ}$ & 1 \\
\hline & $\lfloor-56.112$ & -20 & -50.579 & & \\
\hline & {$[68.191$} & 96 & $67.483]$ & & \\
\hline \multirow[t]{3}{*}{3} & -94.75 & 34.641 & 0 & $0^{\circ}$ & 1 \\
\hline & 10.86 & -20 & 108.28 & & \\
\hline & {$[-31.57$} & 96 & $67.483]$ & & \\
\hline \multirow[t]{2}{*}{4} & 43.48 & 34.641 & 0 & $0^{\circ}$ & 1 \\
\hline & 26.01 & -20 & 108.28 & & \\
\hline
\end{tabular}

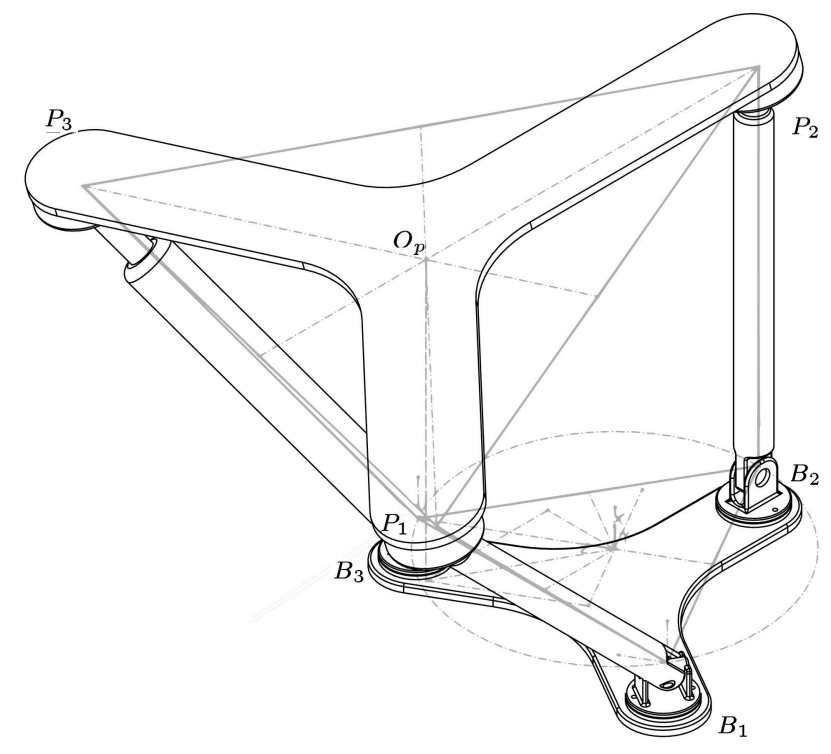

Figure 5. CAD model for geometry variation.

ing that the robot is limited to one of the three zones. In Figure 6(b), the dimensions of the base platform are increased to become equal to the moving platform maintaining the same lengths of the legs; the result is a small zone above the sphere. In Figure 6(c), the dimensions of the base platform are slightly increased to become bigger than those of the moving platform. As a result, the workspace obtained is smaller than that in the previous configuration. In Figure 6(d), the dimensions of the base and moving platforms are the same as in the case of study; however the lengths of the legs are heavily increased, obtaining a bigger continuous workspace, almost $1 / 8$ of the total volume of the sphere. The results show that the workspace is highly sensitive to the changes of the dimensions of the robot; thus, it is encouraged to make an optimization process on the robot to obtain the bigger continuous workspace possible.

\section{Conclusion}

A novel US-RS-RPS 2-DOF parallel robot was presented for different applications with positioning and orientation requirements with a simple actuation. The robot structure as well as the coordinate frames were described, and its kinematic was shown as a combination of translation and rotation. Forward kinematics were solved using the homogeneous transformation matrices and geometrical constraints on moving platform achieving a closed form solution. The use of homogeneous transformation matrices was demonstrated to be a useful and intuitive tool to develop the kinematic of parallel robots, because every member can be analyzed as an open kinematic chain that can be further constrained. The system of equation was solved using the Sylvesters dialytic elimination method and a fourth-degree polynomial was found (representing four possible assembly modes). Inverse 


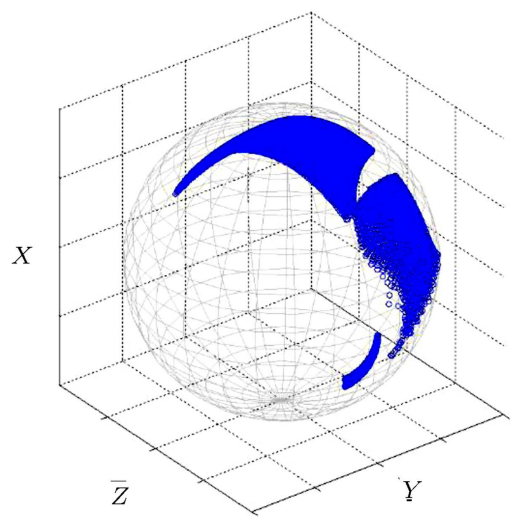

(a)

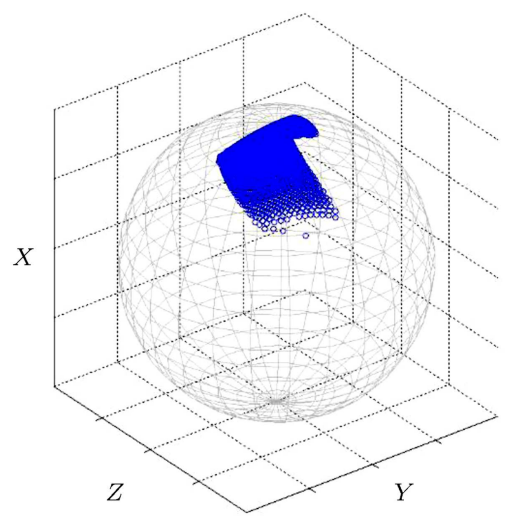

(c)

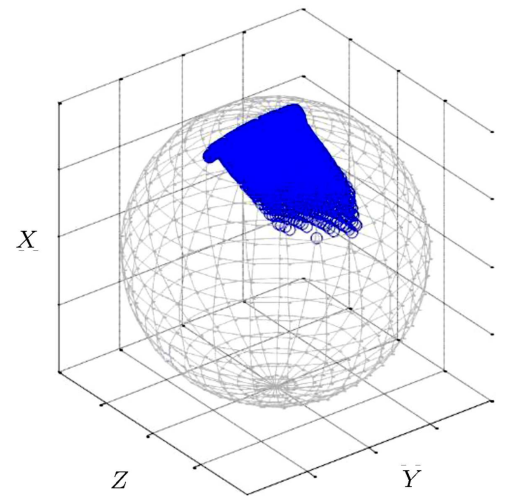

(b)

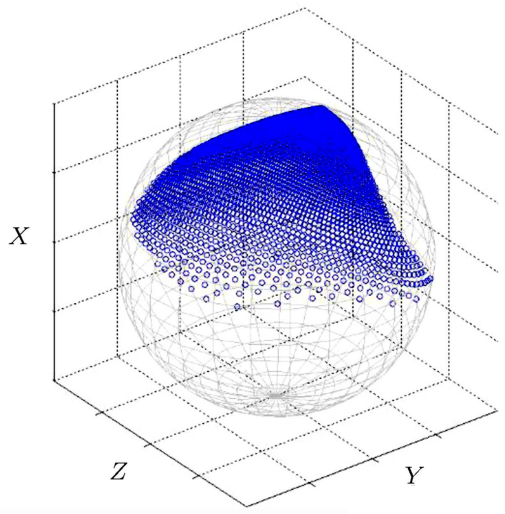

(d)

Figure 6. Projected workspace for different dimensions: (a) $L=96 \mathrm{~mm} d=40 \mathrm{~mm} a=78.5 \mathrm{~mm}$ (case study), (b) $L=96 \mathrm{~mm} d=58 \mathrm{~mm} a=58 \mathrm{~mm}$ (base equal to moving platform), (c) $L=100 \mathrm{~mm} d=69 \mathrm{~mm} a=58 \mathrm{~mm}$ (base bigger than moving platform), and (d) $L=288 \mathrm{~mm} d=40 \mathrm{~mm} a=78.5 \mathrm{~mm}$ (legs dimensions increased).

kinematics was also solved in a straightforward way using matrix homogeneous matrices. A case study was also developed; a comparison of results for direct and inverse kinematics with those of a 3D CAD model shows the effectiveness of the proposed model. Finally, workspace calculation was performed with respect to different geometrical parameters, and showing that the systems workspace is highly influenced by each parameter and configuration.

Future works may extend the current results to the exploration of reconfiguration capabilities based on possible assembly modes to maximize workspace, condition index, and dynamic performance among others, according to user's needs.

\section{References}

1. Fassi, I. and Wiens, G.J. "Multiaxis machining: PKMs and traditional machining centers", Journal of Manufacturing Processes, 2, pp. 1-14 (2000).

2. Taghirad, H.D., Parallel Robots: Mechanics and Control, CRC Press (2013).
3. Merlet, J.-P., Parallel Robots, Springer, Netherlands (2006).

4. Merlet, J.-P. "Direct kinematics of parallel manipulators", IEEE Transactions on Robotics and Automation, 9, pp. 842-846 (1993).

5. Bonev, I., Ilian A., and Gosselin, C.M. "Analytical determination of the workspace of symmetrical spherical parallel mechanisms", IEEE Transactions on Robotics, 22, pp. 1011-1017 (2006).

6. Sadjadian, H., Member, H.T., and Fatehi, A. "Neural networks approaches for computing the forward kinematics of a redundant parallel manipulator", International Journal of Computer, Electrical, Automation, Control and Information Engineering, 2, pp. 1664-1671 (2008).

7. Sadjadian, H. and Taghirad, H. "Comparison of different methods for computing the forward kinematics of a redundant parallel manipulator", Journal of Intelligent and Robotic Systems: Theory and Applications, 44, pp. 225-246 (2005).

8. Jamwal, P.K., Xie, S.Q., Tsoi, Y.H., and Aw, K.C. "Forward kinematics modelling of a parallel ankle rehabilitation robot using modified fuzzy inference", 
Mechanism and Machine Theory, 45, pp. 1537-1554 (2010).

9. Uchida, T. and McPhee, J. "Using Gröbner bases to generate efficient kinematic solutions for the dynamic simulation of multi-loop mechanisms", Mechanism and Machine Theory, 52, pp. 144-157 (2012).

10. Abbasnejad, G., Daniali, H.M., and Fathi, A. "Closed form solution for direct kinematics of a 4PUS 1PS parallel manipulator", Scientia Iranica, 19, pp. 320326 (2012).

11. Lu, Y., Shi, Y., and Hu, B. "Solving reachable workspace of some parallel manipulators by computeraided design variation geometry", Proceedings of the Institution of Mechanical Engineers, 222, pp. 17731782 (2008).

12. Enferadi, J. and Shahi, A. "On the position analysis of a new spherical parallel robot with orientation applications", Robotics and Computer-Integrated Manufacturing, 37, pp. 151-161 (2016).

13. Varedi, S.M., Daniali, H.M., and Ganji, D.D. "Kinematics of an offset 3-UPU translational parallel manipulator by the homotopy continuation method", Nonlinear Analysis: Real World Applications, 10, pp. 1767-1774 (2009).

14. Huang, X.H.X. and He, G.H.G. "New and efficient method for the direct kinematic solution of the general planar Stewart platform", IEEE International Conference on Automation and Logistics, pp. 1979-1983 (2009).

15. Dhingra, A.K., Almadi, A.N., and Kohli, D. "A Gröbner-Sylvester hybrid method for closed-form displacement analysis of mechanisms", Journal of $\mathrm{Me}$ chanical Design, 122, pp. 431-438 (2000).

16. Dash, A.K., Chen, I.M., Yeo, S.H., and Yang, G. "Workspace generation and planning singularity-free path for parallel manipulators", Mechanism and $M a$ chine Theory, 40, pp. 776-805 (2005).

17. Dunlop, G.R. and Jones, T.P. "Position analysis of a two DOF parallel mechanism ]the Canterbury tracker", Mechanism and Machine Theory, 34, pp. 599-614 (1999).

18. Zhang, C. and Zhang, L. "Kinematics analysis and workspace investigation of a novel 2-DOF parallel manipulator applied in vehicle driving simulator", Robotics and Computer-Integrated Manufacturing, 29, pp. 113-120 (2013).

19. Cammarata, A. "Optimized design of a largeworkspace 2-DOF parallel robot for solar tracking systems", Mechanism and Machine Theory, 83, pp. 175-186 (2015).
20. Chaparro-Rico, B. and Castillo-Castaneda, E. "Design of a $2 \mathrm{DOF}$ parallel mechanism to assist therapies for knee rehabilitation", Ingeniería e Investigación, 36, pp. 98-104 (2016).

21. Kucuk, S. and Bingul, Z. "Inverse kinematics solutions for industrial robot manipulators with offset wrists", Applied Mathematical Modelling, 38, pp. 1983-1999 (2014).

22. Zhao, J.-S., Zhou, K., and Feng, Z.-J. "A theory of degrees of freedom for mechanisms", Mechanism and Machine Theory, 39, pp. 621-643 (2004).

23. Zhao, J.-S., Feng, Z.-J., and Dong, J.-X. "Computation of the configuration degree of freedom of a spatial parallel mechanism by using reciprocal screw theory", Mechanism and Machine Theory, 41, pp. 1486-1504 (2006).

24. Shmakov, S.L. "A universal method of solving quartic equations", International Journal of Pure and Applied Mathematics, 71, pp. 251-259 (2011).

25. Lu, Y. "Using CAD functionalities for the kinematics analysis of spatial parallel manipulators with 3-, 4-, 5-, 6-linearly driven limbs", Mechanism and Machine Theory, 39, pp. 41-60 (2004).

26. Lukanin, V. "Inverse Kinematics, Forward Kinematics and working space determination of 3DOF parallel manipulator with SPR Joint Structure", Periodica Polytechnica, 49, pp. 39-61 (2005).

\section{Appendix}

Detailed expressions for $K_{1}$ to $K_{11}$ of Eqs. (9), (10) and (11) are as follows:

$$
\begin{aligned}
K_{1}= & 2 L^{2}-\left(P_{1} P_{2}\right)^{2}+2 d(d-L s \beta)\left(1-c\left(\lambda_{1}-\lambda_{2}\right)\right), \\
K_{2}= & -2 L\left(d-d c\left(\lambda_{1}-\lambda_{2}\right)+L s \beta c\left(\lambda_{1}-\lambda_{2}\right)\right), \\
K_{3}= & -2 L^{2} c \beta, \\
K_{4}= & -2 L s\left(\lambda_{1}-\lambda_{2}\right)(d-L s \beta), \\
K_{5}= & L^{2}\left(1+\rho^{2}\right)+2 d(d-L s \beta)\left(1-c\left(\lambda_{2}-\lambda_{3}\right)\right) \\
& -\left(P_{2} P_{3}\right)^{2}, \\
K_{6}= & -2 \rho L^{2} c \beta, \\
K_{7}= & -2 \rho L\left(d-d c\left(\lambda_{2}-\lambda_{3}\right)+L s \beta c\left(\lambda_{2}-\lambda_{3}\right)\right), \\
K_{8}= & L^{2}-\left(P_{1} P_{3}\right)^{2}+2 d^{2}\left(1-c\left(\lambda_{1}-\lambda_{3}\right)\right)+\rho^{2} L^{2} \\
& +L d \rho s\left(\lambda_{1}-\lambda_{3}-\psi\right)+L d \rho s\left(\lambda_{1}-\lambda_{3}+\psi\right),
\end{aligned}
$$




$$
\begin{aligned}
K_{9}= & -2 L\left(d-d c\left(\lambda_{1}-\lambda_{3}\right)+\operatorname{l\rho s}\left(\lambda_{1}-\lambda_{3}+\psi\right) / 2\right. \\
& \left.-L \rho s\left(\lambda_{1}-\lambda_{3}-\psi\right) / 2\right), \\
K_{10}= & -2 \rho L^{2} c \psi, \\
K_{11}= & -2 L s\left(\lambda_{1}-\lambda_{3}\right)(d-\rho L s \psi) .
\end{aligned}
$$

Detailed expressions for $K_{12}$ to $K_{17}$ of Eq. (13) and (14) are as follows:

$$
\begin{aligned}
& K_{12}=K_{1}-\left(K_{2} s \theta+K_{3} c \theta\right), \\
& K_{13}=2 K_{4}, \\
& K_{14}=K_{1}+\left(K_{2} s \theta+K_{3} c \theta\right), \\
& K_{15}=K_{8}-\left(K_{9} s \theta+K_{10} c \theta\right), \\
& K_{16}=2 K_{11}, \\
& K_{17}=K_{8}+\left(K_{9} s \theta+K_{10} c \theta\right) .
\end{aligned}
$$

Detailed expressions for $K_{18}$ to $K_{22}$ of Eq. (17) are as follows:

$$
\begin{aligned}
& K_{18}=W_{1}+W_{2}, \\
& K_{19}=-4 * W_{4}, \\
& K_{20}=2 W_{1}-2 W_{2}+4 W_{3}, \\
& K_{21}=4 W_{4}, \\
& K_{22}=W_{1}+W_{2},
\end{aligned}
$$

where the $W_{i}$ coefficients are described below:

$$
\begin{aligned}
& W_{1}=4\left(K_{1} K_{11}-K_{4} K_{8}\right)^{2}, \\
& W_{2}=4\left(K_{1} K_{10}-K_{3} K_{8}+K_{3} K_{11}-K_{4} K_{10}\right)
\end{aligned}
$$

$$
\begin{aligned}
& \left(K_{1} K_{10}-K_{3} K_{8}-K_{3} K_{1} 1+K_{4} K_{9}\right), \\
W_{3}= & 4\left(K_{1} K_{9}-K_{2} K_{8}+K_{2} K_{11}-K_{4} K_{9}\right) \\
& \left(K_{1} K_{9}-K_{2} K_{8}-K_{2} K_{11}+K_{4} K_{10}\right), \\
W_{4}= & 4\left(K_{2} K_{3} K_{8}^{2}-K_{2} K_{3} K_{11}^{2}+K_{1}^{2} K_{9} K_{10}\right. \\
& -K_{4}^{2} K_{9} K_{10}-K_{1} K_{2} K_{8} K_{10}-K_{1} K_{3} K_{8} K_{9} \\
+ & \left.K_{2} K_{4} K_{10} K_{11}+K_{3} K_{4} K_{9} K_{11}\right) .
\end{aligned}
$$

\section{Biographies}

Javier Sanjuan acquired BS and MS degrees in Mechanical Engineering from Universidad del Norte, Barranquilla, Colombia in 2012 and 2016, respectively. At present, he is pursuing a scholarship to continue his studies in the field of robotics and control. His main research interests are parallel robots dynamics, design and control.

David Serje Martínez received BS and MS degrees in Mechanical Engineering from Universidad del Norte, Barranquilla, Colombia in 2009 and 2010, respectively. Currently, he is pursuing a PhD degree from the same university thanks to a research grant from Colciencias $\mathrm{PhD}$ National Scholarship. His main research interest are design engineering, machinery modelling and simulation and micro-machining.

Jovanny Pacheco is currently an Assistant Professor in Mechanical Engineering Department in Universidad del Norte (Barranquilla-Colombia). He received his BS degree in Mechanical Engineering in 1998 and also an MSc degree in 2003 in Mechanical Engineering from Universidad del Norte. He also received his PhD degree in Engineering Science from ITESM, Monterrey Mexico. His current research interests are dynamics and synthesis of parallel mechanism, robotics, and micro machining. 